\title{
Cortical brain responses to semantic incongruity and syntactic violation in Italian language: an event-related potential study
}

\author{
Alessandro Angrilli ${ }^{a}{ }^{*}$, Barbara Penolazzi $^{a}$, Francesco Vespignani ${ }^{a}$, Marica De \\ Vincenzi ${ }^{b}$, Remo Job ${ }^{c}$, Laura Ciccarellic, Daniela Palomba ${ }^{a}$, Luciano Stegagno ${ }^{a}$ \\ ${ }^{a}$ Department of General Psychology, University of Padova, Via Venezia 8, 35131 Padova, Italy \\ ${ }^{b}$ University of Chieti and Institute of Psychology of the CNR, Rome, Italy \\ ${ }^{c}$ Department of Developmental and Social Psychology, University of Padova, Padova, Italy
}

Received 5 September 2001; received in revised form 19 November 2001; accepted 26 November 2001

\begin{abstract}
The present experiment investigated cortical responses of native Italian subjects during reading of short sentences including semantic or morphosyntactic violations. Given the specificity of the Italian language in which the sequencing of words is relatively more free than in English or other languages, we investigated whether syntactic and semantic violations were able to elicit event-related potential (ERP) components similar to those found in other languages. Cortical potentials evoked by the anomalous target word were recorded at frontal, central and parietal electrodes. Results showed that, in Italian, semantic anomaly elicited a negative wave (N400) in the 400-500 ms time-window and syntactic error evoked a slower positive wave (P600) in the 500-700 ms time-window. Syntactic error also evoked a significant left anterior negativity in the 350-450 ms time-window, supporting the view that syntactic processes precedes semantic analysis. Thus, Italian language, notwithstanding its specificity, shows ERPs responses to semantic and syntactic violations, with effects, scalp distribution and latency similar to those found in German, Dutch and English. Results point to a cross-linguistic consistency of the semantic and syntactic ERP components associated with the detection of linguistic anomalies. (C) 2002 Elsevier Science Ireland Ltd. All rights reserved.
\end{abstract}

Keywords: Event related potentials; Electroencephalogram; Italian language; Semantic incongruity; Syntactic violation; P600; Left anterior negativity; N400

Evoked potentials have been successfully used to investigate cortical processes underlying language comprehension. Using paradigms based on word-by-word sentence reading, it has been possible to discover two event-related potential (ERP) components which clearly mark anomalies of semantic and syntactic processing. A relatively early negative wave peaking at about $300-400 \mathrm{~ms}$ typically indicates a semantic incongruity [12], and is modulated by the extent of congruence mismatch [13]. A second component, a relatively slower positive wave, termed P600, characterizes grammar violation within a sentence [17]. Additionally, a third component has been observed less consistently, an earlier negative wave located on the left anterior cortex and termed Left Anterior Negativity (LAN) has been found to be elicited by phrase structure violation $[7,16]$. Behavioral studies showed that the way of communicating

\footnotetext{
* Corresponding author. Tel.: +39-049-8276692; fax: +39-0498276600.

E-mail address: alessandro.angrilli@unipd.it (A. Angrilli).
}

syntactic information differs across languages [2,3]. Furthermore, recent studies performed with functional magnetic resonance imaging and evoked potentials suggest that different cultures and languages are able to activate distinct cortical networks during language elaboration $[1,19]$. However, within the group of Germanic languages, the N400 and the P600, the two most studied components, have been shown to represent consistent landmarks of semantic and syntactic violation, in English [12,17], Dutch [10,11] and German [7,8]. In Italian language, sentence structure is characterized by a less rigid order of words [4]. As an example, these two sentences are equivalent for an Italian speaker: "Mario lo ha detto" and "Lo ha detto Mario" (Mario has told that), while in English or German, only one form is used. Indeed, within a sentence, the probability for the initial noun to be a subject is much higher in English and Dutch than in Italian. Since for Italians, the initial noun is not necessarily the subject, some sentences may become ambiguous. For instance, the 
sentence "Ha chiamato una ragazza" may well be interpreted both as "Someone called a girl" and "A girl called".

The relatively more flexible word order of Italian may affect both the extent and the timing (and therefore the landmark components, such as the N400 and P600) of the electrophysiological responses involved in the detection of semantic and syntactic violations. In particular, given the tolerance for the order of words, the detection of anomalies could be delayed and therefore might induce a time shift of the classical ERP waves. The alternative hypothesis [6] states that the structure of semantic and syntactic analysis of a sentence is universal and therefore should be found in all languages, also in those, like Italian, in which the probabilistic constraints of word order are less rigid and deterministic.

Therefore, the investigation of the electrophysiological correlates of linguistic violation in native Italian speakers would be of interest for both the cross-linguistic aspects of parsing and the timing of the processes involved in semantic and syntactic violations.

In order to test the mentioned hypotheses, we have run an experiment in which subjects were administered sentences with subject-verb semantic violations and subject-verb number agreement morphosyntactic violations ${ }^{1}$, while evoked potentials were recorded. A total of 360 sentences was constructed. One-hundred and twenty sentences served as fillers whereas the remaining 240 sentences were analyzed. Half of these were 'well-formed' (semantically and grammatically correct), half included a violation. In 60 sentences, there was a subject-verb semantic violation, for example: Il bambino impaurito SCAPPA di fronte al cacciatore - Il bambino impaurito PIOVE di fronte al cacciatore (The scared child ESCAPES in front of the hunter - The scared child RAINS in front of the hunter). In 60 sentences, there was a subject-verb number agreement violation, for example: Il vecchio cameriere SERVE con espressione distratta - Il vecchio cameriere SERVONO con espressione distratta (The old waiter SERVES with inattentive expression - The old waiter SERVE with inattentive expression). Each sentence in the violated set was derived from a sentence in the correct set, in a way that the words preceding and following the violation (a verb always in fourth position) were the same as in the companion correct sentence; therefore, the set of incorrect and correct sentences were matched for the number of words (eight for the semantic, seven for the syntactic conditions). The described materials were divided into two lists of 180 sentences each, by paying attention that the members of a pair of incorrect and correct companion sentences were

\footnotetext{
${ }^{1}$ Although the last kind of violation is morphosyntactic rather than pure syntactic, we will refer to this violation as syntactic in order to be more consistent with most ERP literature which has been using very often morphosyntactic tasks. Thus, in ERP investigations, morphosyntactic errors, for the sake of simplicity, are typically described as syntactic errors (see for instance [10-12]).
}

assigned to different lists. The sentences were displayed word-by-word (this method is a must when evoked potentials are recorded), with each word appearing in the center of the screen for $300 \mathrm{~ms}$. The inter-word-interval was $300 \mathrm{~ms}$ and the inter-trial-interval $1500 \mathrm{~ms}$. Subjects were asked to read the sentences for comprehension and randomly, every few sentences, they had to answer some questions (with a 'yes' or a 'no') about their content: this instruction was aimed to force the subject to read sentences carefully. The subjects were warned that some sentences could include errors. ERPs were recorded from 19 scalp electrodes placed according to the International 10-20 System and referred to linked mastoids. Vertical and horizontal eye movements were monitored through four electrodes placed around the orbital region (bipolar montage). ERPs and electrooculogram signals were amplified with a SynAmp system (Neuroscan system), with DC - $100 \mathrm{~Hz}$ bandpass, and were digitized continuously with a sampling rate of 500 Hz. Twenty-five native Italian-speaking university students (12 males, 13 females; age range, 18-29 years), all righthanded, participated in the experiment. The EEG artifacts due to vertical eye movements and blinks were corrected by using VEOG (Vertical Electroculogram) channels as reference, and next data were epoched in the interval -200/1200 ms with respect to the onset of the target word. Epochs including residual artifacts exceeding a $150 \mu \mathrm{V}$ amplitude were visually inspected and rejected, and the accepted epochs were averaged. Analysis was focused on the violated target word, which was always in the 4th position. For statistical analysis, the Electrode group variable consisted of the following levels: Frontal (F3, Fz, F4); Central (C3, $\mathrm{Cz}, \mathrm{C} 4)$; and Parietal (P3, Pz, P4). A further variable was kind of Sentence (Control vs. Violation). The time-windows for the semantic and syntactic violations were a priori chosen according to the latency of the classical components N400 (time-window 400-500 ms) and P600 (time-window 500-700 ms).

Semantic violation elicited a negative wave (N400) that reached the maximum over centro-parietal electrodes at about $450 \mathrm{~ms}$ (Fig. 1, upper panel, dashed line). In line with this observation, statistical analysis (analysis of variance) showed a significant main effect of Sentence $\left(F_{1,24}=5.75, P<0.02\right)$ in the $400-500 \mathrm{~ms}$ interval, with greater negativity to violation than to the control condition (Fig. 2, upper panel). The analysis of the 500-700 ms interval did not reveal any effect $\left(F_{1,24}=1.01\right.$, n.s. $)$. These results are consistent with the scalp distribution and latency of the N400 elicited by semantic incongruity in other languages such as English, Dutch and German [8,11,12], and point to the universal cross-linguistic reliability of the $\mathrm{N} 400$ as an index of semantic incongruity within the sentence.

Sentences with syntactic errors (number-verb disagreement) showed a clear positivity, in the target word, starting at about $500 \mathrm{~ms}$ and sustained until $700 \mathrm{~ms}$ (Fig. 1, lower panel, dashed line). The observed positivity was more 

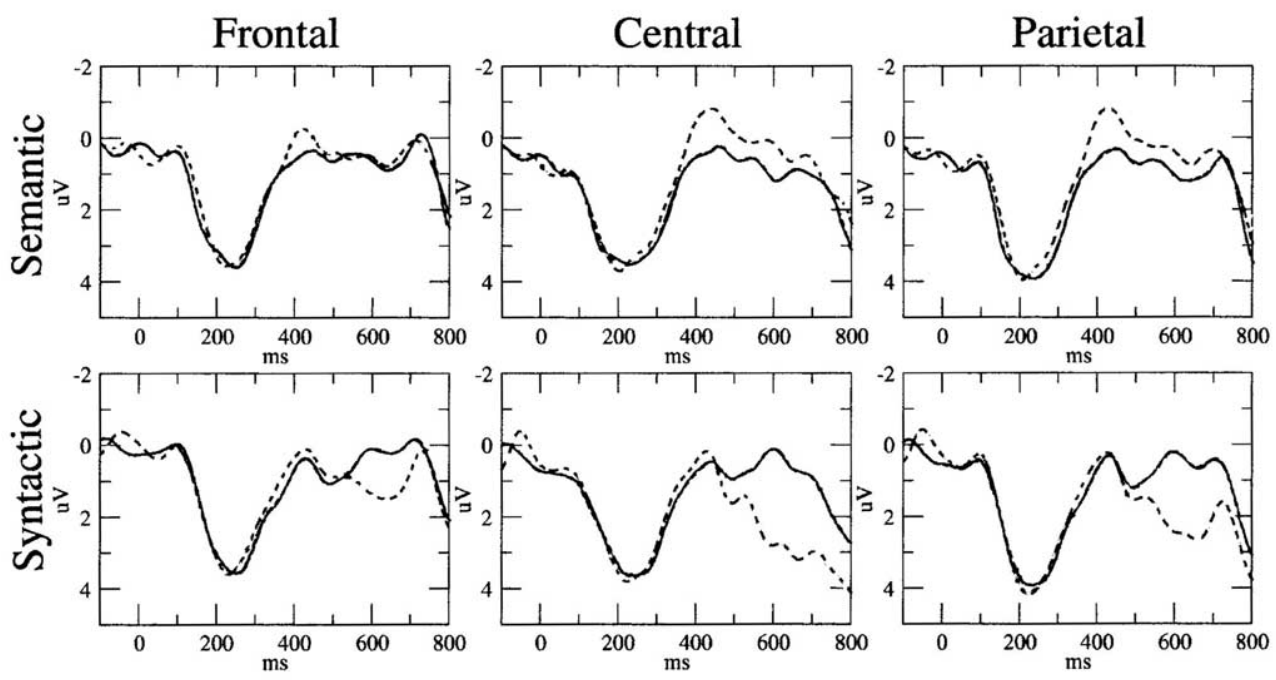

Fig. 1. Grand-average waveforms representing $800 \mathrm{~ms}$ of potentials evoked by the target word, a verb in the fourth position, in semantic (upper panel) and syntactic (lower panel) sentences. The waveform of the word in the anomalous sentence is a dashed line whereas the word in the correct phrase is a full line. Waveforms represent the average of three electrodes located in the frontal, central and parietal sites.

pronounced over central and parietal sites. Statistics computed for the 400-500 ms interval did not produce any significance, whereas in the 500-700 ms interval, there was a main effect of Sentence $\left(F_{1,24}=5.05\right.$, $P<0.03)$ with violation significantly more positive than the control condition (Fig. 2, lower panel). Also, this result is consistent with several experiments performed in other languages $[7,10,11,17]$, and confirms the P600 as a component associated with syntactic errors.

A further analysis was done to test the hypothesis that syntactic violation evokes also an early LAN. The mean amplitude of four fronto-central electrodes placed over left hemisphere (which are: T3; C3; F3; F7) in the 350-450 ms time-window was computed. A one-tailed (we expected greater negativity to the violation) $t$-test for dependent samples was used to compare syntactic violation with control. The statistical result indicates a significant $\left(t_{23}=1.86, P<0.04\right.$, one-tailed) relative negativity to syntactic violation as compared with the control condition (difference, $-0.71 \mu \mathrm{V}$ ). This result is in line with data obtained from other languages $[7,16]$, but also with the hypothesis, in both the theoretical and experimental literature, that the detection of syntactic anomalies should happen earlier than semantic ones $[5,20]$. The localization of the LAN in the left anterior cortex, found in our as well as in other investigations here quoted, is consistent with the observation, through positron emission tomography measures, of the activation of Broca's area during syntactic and morphosyntactic violations [15].

Some authors [9] suggested that the difficulty in obtaining a consistent LAN across studies may depend on partial overlapping with exogenous components evoked by the physical characteristics of the linguistic stimuli (such as the visual
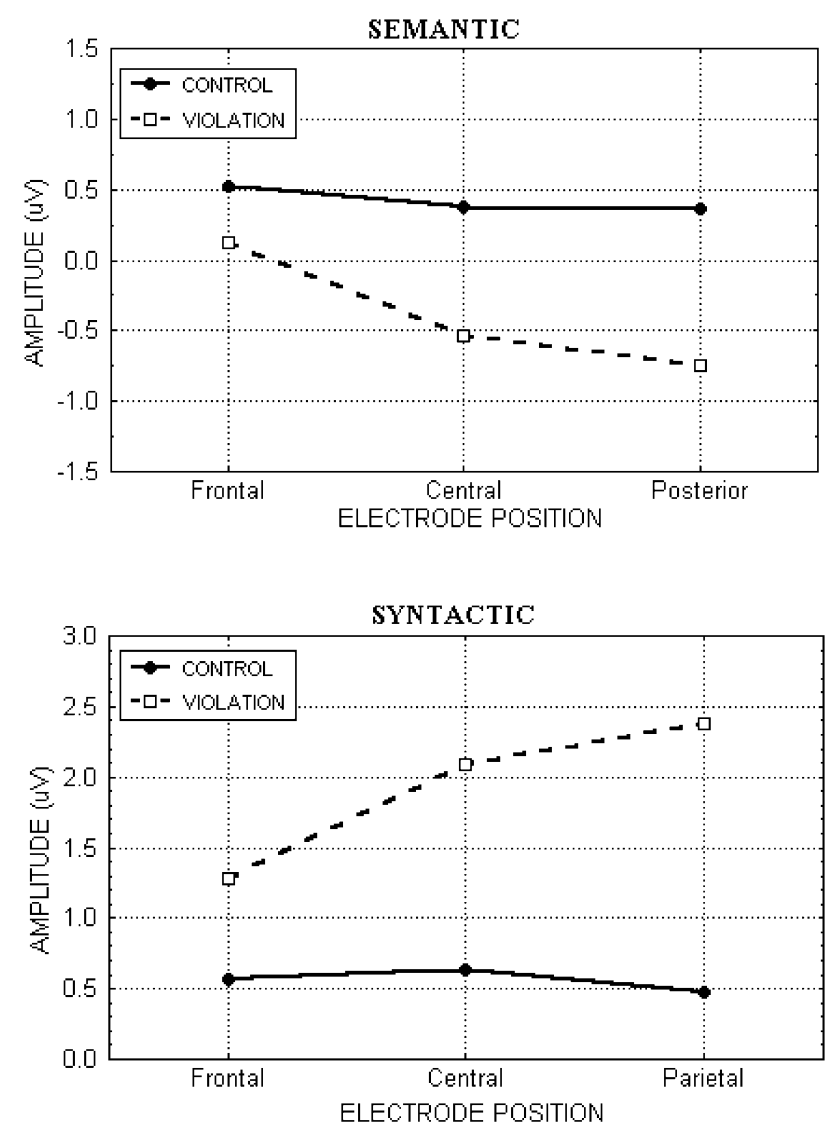

Fig. 2. Graphs representing the mean values obtained from significant statistics at three electrode locations, frontal, central and parietal. Data are relative to the $400-500 \mathrm{~ms}$ time-window for semantic incongruity (upper panel), and 500-700 ms timewindow for syntactic error (bottom panel). 
contrast of the words). Another relevant physical parameter which may play against a significant LAN effect (but also on the different latency of LAN found in literature) is word length. A significant positive correlation has been found between LAN latency and word length [18]. Thus, if the sample of words selected as stimuli for an experiment is characterized by large length variability, any early linguistic process (such as detection of syntactic error) may be underestimated because of phase shift of the LAN components across trials.

Our electrophysiological results are in line with some theories on sentence comprehension which postulate that the syntactic analysis is distinct from the semantic analysis, and this should hold in all languages (see, for instance [6]). The alternative theory, which here we discarded, claims that syntactic and semantic analyses interact directly and simultaneously and, furthermore, that their relative weight depends on probabilistic constraints that may vary across languages [14]. According to the last model, a language like Italian, which is characterized by a less rigid word order [4], and in which therefore the semantic analyzer should be more important than the syntactic one for sentence comprehension ${ }^{2}$, should elicit electrophysiological processes and components (such as N400, P600 and LAN) different from languages with more rigid word order constraints.

In conclusion, despite the specific differences between Italian and the other languages, in our study, we found that semantic incongruence and syntactic violation elicited electrophysiological components very similar, for both scalp distribution and the latency, to those found in several other languages. The Italian language, with its peculiarities, is a useful tool to test some models on sentence comprehension, and this will be the aim of future research more oriented to theoretical questions.

[1] Angrilli, A., Dobel, C., Rockstroh, B., Stegagno, L. and Elbert, T., EEG brain mapping of phonological and semantic tasks in Italian and German languages, Clin. Neurophysiol., 111 (2000) 706-716.

[2] Bach, E., Brown, C. and Marslen-Wilson, W.D., Crossed and nested dependencies in German and Dutch: a psycholinguistic study, Lang. Cogn. Process., 1 (1986) 249-262.

[3] Cuetos, F. and Mitchell, D.G., Cross linguistic differences in parsing: restrictions on the use of the late closure strategy in Spanish, Cognition, 30 (1988) 73-105.

\footnotetext{
${ }^{2}$ In a language with a relatively free word order, the detection of syntactic anomalies would be less efficient because of word order tolerance, whereas the semantic analyzer would be strengthened and possibly it would support both the syntactic and morphological analyzers in error detection.
}

[4] De Vincenzi, M., Syntactic analysis in sentence comprehension: effects of dependency types and grammatical constraints, J. Psycholinguist. Res., 25 (1996) 117-133.

[5] Forster, K.I., Levels of processing and the structure of the language processor, In W. Cooper and E.C.T. Walker (Eds.), Sentence Processing, Erlbaum, Hillside, NJ, 1979.

[6] Frazier, L. and Rayner, K., Making and correcting errors during sentence comprehension: eye movements in the analysis of structurally ambiguous sentences, Cogn. Psychol., 14 (1982) 178-210.

[7] Friederici, A.D. and Mecklinger, A., Syntactic parsing as revealed by brain responses: first-pass and second-pass parsing processes, J. Psycholinguist. Res., 25 (1996) 157176.

[8] Friederici, A.D., Steinhauer, K. and Frish, S., Lexical integration: sequential effects of syntactic and semantic information, Mem. Cognit., 27 (1999) 438-453.

[9] Gunter, T.C., Friederici, A.D. and Hahne, A., Brain responses during sentence reading: visual input affects central processes, NeuroReport, 10 (1999) 3175-3178.

[10] Hagoort, P. and Brown, C., ERP effects of listening to speech compared to reading: the P600/SPS to syntactic violations in spoken sentences and rapid serial visual presentation, Neuropsychologia, 38 (2000) 1531-1549.

[11] Hagoort, P., Brown, C. and Groothusen, J., The syntactic positive shift as an ERP measure of syntactic processing, Lang. Cogn. Process., 8 (1993) 439-484.

[12] Kutas, M. and Hillyard, S.A., Event-related brain potentials to grammatical errors and semantic anomalies, Mem. Cognit., 11 (1983) 539-550.

[13] Kutas, M. and Hillyard, S.A., Brain potentials during reading reflect word expectancy and semantic association, Nature, 307 (1984) 161-163.

[14] MacDonald, M.C., Pearlbutter, N.G. and Seidenberg, M.S., The lexical nature of syntactic ambiguity resolution, Psychol. Rev., 101 (1994) 676-703.

[15] Moro, A., Tettamanti, M., Perani, D., Donati, C., Cappa, S.F. and Fazio, F., Syntax and the brain: disentangling grammar by selective anomalies, Neuroimage, 13 (2001) 110-118.

[16] Neville, H.J., Nicol, J.L., Barss, A., Forster, K.I. and Garret, M.F., Syntactically based sentence processing classes: evidence from event-related brain potentials, J. Cogn. Neurosci., 3 (1991) 151-165.

[17] Osterhout, L. and Holcomb, P.J., Event-related brain potentials, elicited by syntactic anomaly, J. Mem. Lang., 31 (1992) 785-806.

[18] Osterhout, L., Bersick, M. and McKinnon, R., Brain potentials elicited by words: word length and frequency predict the latency of an early negativity, Biol. Psychol., 46 (1997) 143-168.

[19] Paulesu, E., McCrory, E., Fazio, F., Menoncello, L., Brunswick, N., Cappa, S.F., Cotelli, M., Cossu, G., Corte, F., Lorusso, M., Pesenti, S., Gallagher, A., Perani, D., Price, C., Frith, C.D. and Frith, U., A cultural effect on brain function, Nat. Neurosci., 3 (2000) 91-96.

[20] Rayner, K., Garrod, S. and Perfetti, C.A., Discourse influences during parsing are delayed, Cognition, 45 (1992) 109-139. 